\title{
Laboratory astrophysics with laser-driven strong magnetic fields in China
}

Fei-Lu Wang ${ }^{1}$, Xiao-Xing Pei ${ }^{1,6}$, Bo Han ${ }^{1,6}$, Hui-Gang Wei ${ }^{1}$, Da-Wei Yuan ${ }^{1}$, Gui-Yun Liang ${ }^{1}$, Gang Zhao ${ }^{1,6}$, Jia-Yong Zhong ${ }^{2}$, Zhe Zhang ${ }^{3}$, Bao-Jun $\mathrm{Zhu}^{3,6}$, Yan-Fei $\mathrm{Li}^{3,6}$, Fang $\mathrm{Li}^{3}$, Yu-Tong $\mathrm{Li}^{3,6}$, Si-Liang Zeng ${ }^{4}$, Shi-Yang Zou ${ }^{4}$, and Jie Zhang ${ }^{5}$

${ }^{1}$ Key Laboratory of Optical Astronomy, National Astronomical Observatories, Chinese Academy of Sciences, Beijing 100012, China

${ }^{2}$ Department of Astronomy, Beijing Normal University, Beijing 100875, China

${ }^{3}$ Beijing National Laboratory of Condensed Matter Physics, Institute of Physics, Chinese Academy of Sciences, Beijing 100190, China

${ }^{4}$ Institute of Applied Physics and Computational Mathematics, Beijing, 100088, China

${ }^{5}$ Shanghai Jiao Tong University, Shanghai 200240, China

${ }^{6}$ University of Chinese Academy of Sciences, Beijing 100049, China

(Received 30 March 2016; revised 7 July 2016; accepted 12 July 2016)

\begin{abstract}
In this paper, the recent studies of laboratory astrophysics with strong magnetic fields in China have been reviewed. On the Shenguang-II laser facility of the National Laboratory on High-Power Lasers and Physics, a laser-driven strong magnetic field up to $200 \mathrm{~T}$ has been achieved. The experiment was performed to model the interaction of solar wind with dayside magnetosphere. Also the low beta plasma magnetic reconnection (MR) has been studied. Theoretically, the model has been developed to deal with the atomic structures and processes in strong magnetic field. Also the study of shock wave generation in the magnetized counter-streaming plasmas is introduced.
\end{abstract}

Keywords: Laboratory astrophysics; laser; strong magnetic fields

\section{Magnetic-field generation in the laboratory}

Laboratory generation of strong magnetic fields is of significance to many research fields including plasma and beam physics ${ }^{[1]}$, astrophysics ${ }^{[2]}$, material science ${ }^{[3]}$, and atomic and molecular physics ${ }^{[4]}$, and so forth. In the aspect of astrophysics, a high magnetic field is related to many high-energy astrophenomena, such as the jet formation ${ }^{[5]}$, magnetic reconnection $(\mathrm{MR})^{[6,7]}$ and collisionless shock formations. Laboratory generation of strong magnetic fields makes it possible to study them in a new regime and controllable way.

A laser-driven strong magnetic field up to $200 \mathrm{~T}$ has been demonstrated on the Shenguang-II (SG-II) laser facility of the National Laboratory on High-Power Lasers and Physics. The basic scheme is to produce strong magnetic fields

Correspondence to: G. Zhao, Key Laboratory of Optical Astronomy, National Astronomical Observatories, Chinese Academy of Sciences, Beijing 100012, China, Email: gzhao@ bao.ac.cn; Jie Zhang, Shanghai Jiao Tong University, Shanghai 200240, China, Email: jzhang1@sjtu.edu.cn; Yutong Li, Beijing National Laboratory of Condensed Matter Physics, Institute of Physics, Chinese Academy of Sciences, Beijing 100190, China, Email: ytli@iphy.ac.cn from the cold electron flow in a laser irradiated open-ended coil $^{[8]}$. Compared with the previous generation of magnetic fields driven by fast electron current in a capacitor-coil target $^{[9]}$, the generation mechanism of the magnetic field is straightforward and the coil is easy to be fabricated.

The experiment layout is shown in Figure 1. Eight SGII beams with total energy of $2 \mathrm{~kJ}$ in $1 \mathrm{~ns}$ were focused on a planar plate attached on the end of the open-ended coil. During the laser irradiating the planar plate, fast electrons were generated and expelled out from the laser focal spot. This would lead to a high electrostatic potential grown up near the laser focus and charged the target. The potential dragged the background cold electrons to the focus to neutralize the target. This cold electron current in the target coil would create a strong magnetic-field pulse. The strength of the magnetic field was measured by a precalibrated B-dot.

The laser parameters, magnetic-field strength, current and energy conversion efficiency, are all summarized in Table 1. The field strength at the coil center, $B_{\text {center, }}$ is generally increases as a function of $I \lambda^{2}$. The $B_{\text {center reaches a }}$ 
Table 1. Summary of magnetic-field strength, and current in the coil.

\begin{tabular}{|c|c|c|c|c|c|c|}
\hline $\begin{array}{l}E_{\text {laser }} \\
\text { (J) }\end{array}$ & $\begin{array}{l}\text { Wavelength } \\
(\mathrm{nm})\end{array}$ & $\begin{array}{l}I \lambda^{2} \\
\left(\mathrm{~W} \mathrm{~cm}^{-2} \mu \mathrm{m}^{2}\right)\end{array}$ & Method & $\begin{array}{l}\text { Magnetic-field strength at the B-dot } \\
\text { (T) }\end{array}$ & $\begin{array}{l}\text { Current } \\
(\mathrm{mA})\end{array}$ & $\begin{array}{l}B_{\text {center }} \\
\text { (T) }\end{array}$ \\
\hline 536.24 & 351 & $1.87 \times 10^{14}$ & B-dot & 0.0065 & 0.044 & 45 \\
\hline 1004.27 & 351 & $3.50 \times 10^{14}$ & B-dot & 0.011 & 0.071 & 73 \\
\hline 1780.4 & 351 & $6.21 \times 10^{14}$ & B-dot & 0.16 & 0.10 & 102 \\
\hline 1966.8 & 351 & $6.85 \times 10^{14}$ & B-dot & 0.031 & 0.20 & 205 \\
\hline
\end{tabular}

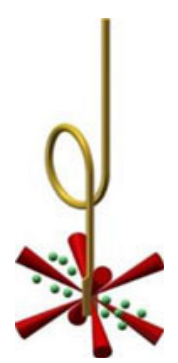

Figure 1. Schematic view of the experimental setup at the Shenguang-II laser facility. Eight laser beams at $351 \mathrm{~nm}$ were divided into two bunches and focused onto both sides of the planar plate simultaneously.

maximum value of $205 \mathrm{~T}$, at the highest $I \lambda^{2}$ of $6.85 \times$ $10^{14} \mathrm{~W} \mathrm{~cm}^{-2} \mu \mathrm{m}^{2}$. The conversion efficiency from laser energy to magnetic field is about $1 \%$ in this experiment.

\section{Magnetic reconnection}

MR is a universal physical process in plasmas, in which the stored magnetic energy is converted into high-velocity flows and energetic particles ${ }^{[10,11]}$. The model of MR is widely applied in astrophysics, including investigations on solar flares ${ }^{[12,13]}$, star formation ${ }^{[14]}$, the coupling of solar wind with earth magnetosphere, accretion disks and Gamma-ray bursts ${ }^{[15]}$, and so forth. The process of MR has been studied in detail by dedicated magnetic-driven experiments ${ }^{[16]}$. Based on the quasi-steady state of the selfgenerated magnetic fields in laser plasmas, we reconstruct the topology of MR and model the loop-top x-ray source ${ }^{[7]}$, the interaction of solar wind with dayside magnetosphere ${ }^{[17]}$ in the laboratory by using SG-II laser facility. The two MR processes modeled in laboratory are shown in Figure 2 as follows.

Figure 2(a) shows a cartoon simulation of the solar flares and the coupling of solar wind with earth magnetosphere. Figure 2(b) shows the x-ray image of laser-driven MR which is designed to be similar to the scheme of a loop-top x-ray source in the solar flares. The reconnection plasma produced on the aluminum $(\mathrm{Al})$ target with two laser beams, and the downward outflow will interact with a preset copper $(\mathrm{Cu})$ target. Two bright $\mathrm{x}$-ray spots are clearly observed due to the laser heating the $\mathrm{Al}$ foil target where two laser spots are (a) (b)

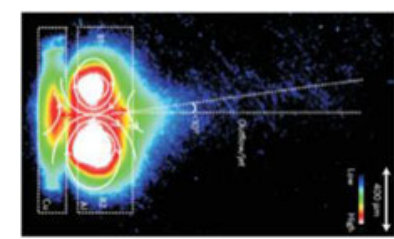

(c)

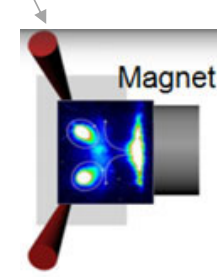

Figure 2. (a) Shows a cartoon simulating the solar flares and the coupling of solar wind with earth magnetosphere. (b) MR model for the loop-top $\mathrm{x}$-ray source, $\mathrm{x}$-ray images taken with Pinhole camera in the experiment. (c) MR model for the interaction of solar wind with magnetosphere, one micro-solar-flare is produced by two intense laser beams interacting with a solid aluminum block, and the outflow interacts with a preset permanent magnetic pole.

separated by $600 \mu \mathrm{m}$. The most striking feature in the image is that a bright $\mathrm{x}$-ray spot at the center of the $\mathrm{Cu}$ target is observed just below the downward outflow/jet. The position and the arc shape of the spot are the solid evidence that high speed outflow/jet on the Al foil impacting the plasma generated on the $\mathrm{Cu}$ target, a picture clearly resembling the loop-top x-ray source in solar flare observations.

Following previous one, another experiment was performed to model the interaction of solar wind with dayside magnetosphere. Figure 2(c) shows the schematic experimental setup and $\mathrm{x}$-ray image for laser-driven MR. One micro-solar-flare ${ }^{[7]}$ is produced by two intense laser beams interacting with a solid aluminum block. The magnetized outflowing plasma is evident between two plasma bubbles and is used to interact with a preset permanent magnetic pole, then further models dynamic processes in Solar-Earth space. Scaling law shows the present study will be helpful for understanding Solar-Earth space dynamic phenomena. The traces of high-energy electrons are also found moving along with the magnetic separatrix. 


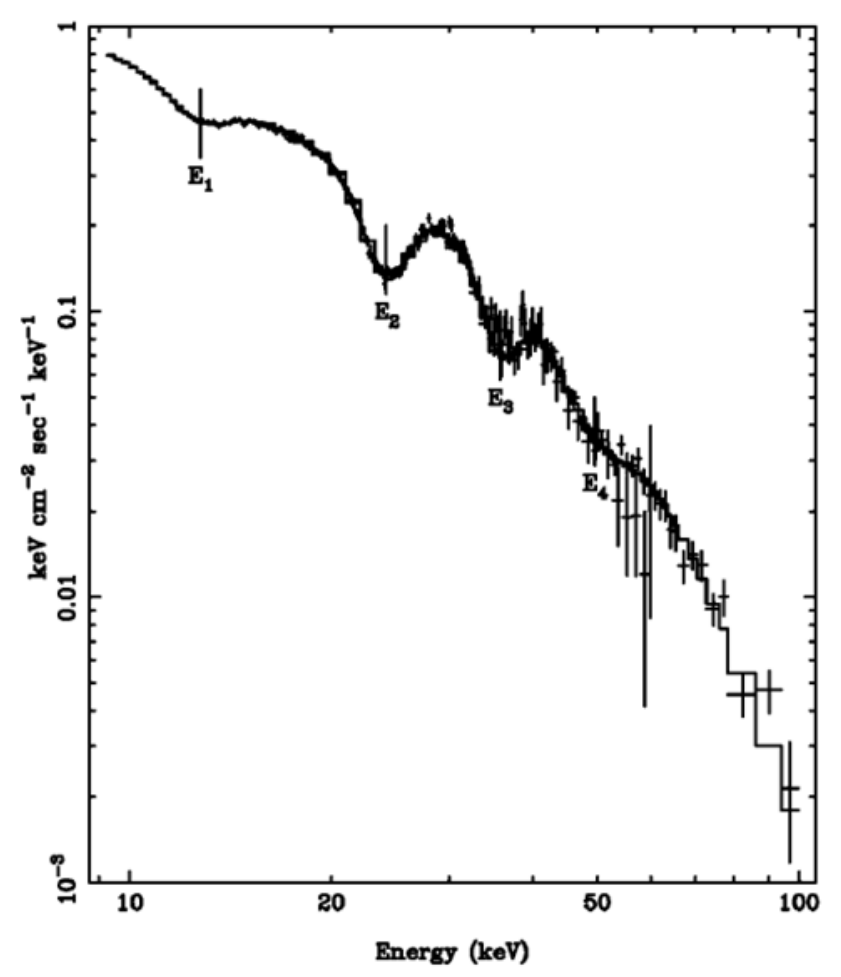

Figure 3. Unfolded spectrum of the descending edge of the main peak of $\mathrm{X} 0115+63^{[2]}$.

Furthermore, we have studied the low beta plasma MR in our recent experiment. This is the first laboratory study of MR with an explicitly controlled magnetic-field environment produced by capacitor-coil target ${ }^{[18]}$. The magnetic field measured in laboratory is about dozens of Tesla. Under such strong magnetic-field environment, we have measured the MR outflow. The result proves indirectly the existence of a reconnection-induced electric field in a low beta MR, which could show potential applications in astrophysics and plasma physics, such as the study of reconnection in solar and magnetotail flares.

\section{Spectra of plasma with strong magnetic field related to astrophysical objects}

Magnetic fields are widely existed in universe, from the interior of stars to the astrophysical interstellar medium (ISM). For example, in $\mathrm{x}$-ray binaries the magnetic-field strengths of accreting neutron stars could be larger than $10^{8} \mathrm{~T}$ by observing the cyclotron features as Figure 3 shows. Here four harmonic features were observed, which are interpreted in terms of the four harmonically spaced lines of cyclotron resonant features in a strong magnetic field ${ }^{[2]}$.

The atomic structures and processes in strong magnetic field have been studied theoretically by various methods, such as finite element ${ }^{[19]}$, Kantorvich ${ }^{[20]}$, semi-classical closed orbit theory ${ }^{[20]}$, finite basis expansion ${ }^{[21]}$ and power series expansion ${ }^{[22]}$. Those calculations of the electron wavefunctions are very complicated and hard to be applied to the combination of B- and E-fields. The coulomb wavefunction discrete variable presentation method (CWDVR) is a simple and highly accurate method of investigating the behavior of atomic system in a strong magnetic field up to $B \sim 2.35 \times 10^{9} \mathrm{~T}^{[23]}$.

Recently, extremely strong magnetic field can be generated using high-power laser in laboratory. Those magnetic fields can be up to thousand Tesla ${ }^{[18]}$. Thus, it is very interesting to investigate the properties of matters related to the astrophysics under such a strong magnetic field. Atomic levels are shifted and split in strong magnetic field, which would certainly introduce changes in the opacity of matters. Measurements of the opacity under strong magnetic field are in progress with SG-II laser facility. Effects of the strong magnetic on opacities will be investigated. The results would benefit on the understanding of the energy transport in astrophysics. It could also be a potential candidate to solve the present problems on the opacity in solar convection zones $^{[24]}$.

\section{Studying shock wave generation in magnetized counter- streaming plasmas}

Energetic particles are ubiquitous in astrophysical plasmas. However, the physical acceleration process is not well understood. The most remarkable example is the solar energetic particles ${ }^{[25]}$. The kinetic energies of the ejected particles are up to $\sim 10^{25} \mathrm{~J}$, which are believed to be the most explosive events in the Solar System. Recently, astro-observation ${ }^{[26]}$ shows that particle acceleration is associated with shock wave, which is driven by the eruption of magnetized plasmoids, called coronal mass ejections (CMEs). Studying the shock wave generation in the magnetized plasmas is important to understand the shock wave acceleration in the Solar System.

The scaled-down and controllable laboratory experiments, as an accessory to the astronomical observations, can closely study the collisionless shock wave using high-power lasers. Counter-streaming plasmas system is a test bed for studying such phenomena in laboratory ${ }^{[27]}$. Previous SG-II experiments have successfully generated shock wave using unmagnetized counter-streaming plasmas ${ }^{[28-30]}$. In order to generate magnetized plasmas to study the shock wave driven by the CMEs, we plan to add a kilo-Tesla level magnetic field induced by the capacitor-coil target ${ }^{[18]}$ into the counterstreaming plasmas system. Assuming the average state of ionization $Z=6$, the velocity of the streams $V=$ $1000 \mathrm{~km} \mathrm{~s}^{-1}$, and the strength of B-field $B=1000 \mathrm{~T}$, the ion cyclotron radius can be estimated as $r \sim 20 \mu \mathrm{m}$, which is smaller than the system scale $L=4.5 \mathrm{~mm}$. The electron 
cyclotron radius is much smaller than the ion's, because of the larger charge-to-mass ratio. The conditions can ensure that it is suitable to study shock wave driven by the magnetized plasmas in laboratory.

\section{Acknowledgments}

This work is supported by National Basic Research Program of China (973 Program) under grant No. 2013CBA01503, and the National Natural Science Foundation of China under grant No. 11573040, 11503041 and 11135012. Also supported by the Science Challenge Program.

\section{References}

1. R. Z. Sagdeev, Rev. Plasma Phys. 4, 23 (1966).

2. A. Santangelo, A. Segreto, S. Giarrusso, D. Dal Fiume, M. Orlandini, A. N. Parmar, T. Oosterbroek, T. Bulik, T. Mihara, S. Campana, G. L. Israel, and L. Stella, Astrophys. J. 523, L85 (1999).

3. E. Kojima, A. Miyata, Y. Motome, H. Ueda, Y. Ueda, and S. Takeyama, J. Low Temp. Phys. 159, 3 (2010).

4. P. Gilch, F. Pollinger-Dammer, C. Musewald, M. E. MichelBeyerle, and U. E. Steiner, Science 281, 982 (1998).

5. R. Albertazzi, A. Ciardi, M. Nakatsutsumi, T. Vinci, J. Beard, R. Bonito, J. Billette, M. Borghesi, Z. Burkley, S. N. Chen, T. E. Cowan, T. Herrmannsdorfer, D. P. Higginson, F. Kroll, S. A. Pikuz, K. Naughton, L. Romagnani, C. Riconda, G. Revet, R. Riquier, H. P. Schlenvoigt, I. Y. Skobelev, A. Y. Faenov, A. Soloviev, M. Huarte-Espinosa, A. Frank, O. Portugall, H. Pepin, and J. Fuchs, Science 346, 325 (2014).

6. S. Masuda, T. Kosugi, H. Hara, and Y. Ogawara, Nature 371, 495 (1994).

7. J. Y. Zhong, Y. T. Li, X. G. Wang, J. Q. Wang, Q. L. Dong, C. J. Xiao, S. J. Wang, X. Liu, L. Zhang, L. An, F. L. Wang, J. Q. Zhu, Y. A. Gu, X. T. He, G. Zhao, and J. Zhang, Nat. Phys. 6, 984 (2010).

8. B. J. Zhu, Y. T. Li, D. W. Yuan, Y. F. Li, F. Li, G. Q. Liao, J. R. Zhao, J. Y. Zhong, F. B. Xue, S. K. He, W. W. Wang, F. Lu, F. Q. Zhang, L. Yang, K. N. Zhou, N. Xie, W. Hong, H. G. Wei, K. Zhang, B. Han, X. X. Pei, C. Liu, Z. Zhang, W. M. Wang, J. Q. Zhu, Y. Q. Gu, Z. Q. Zhao, B. H. Zhang, G. Zhao, and J. Zhang, Appl. Phys. Lett. 107, 261903 (2015).

9. S. Fujioka, Z. Zhang, K. Ishihara, K. Shigemori, Y. Hironaka, T. Johzaki, A. Sunahara, N. Yamamoto, H. Nakashima, T. Watanabe, H. Shiraga, H. Nishimura, and H. Azechi, Sci. Rep. 3, 1170 (2013).
10. M. Yamada, Phys. Plasmas 14, 058102 (2007).

11. E. Zweibel and M. Yamada, Annu. Rev. Astron. Astrophys. 47, 291 (2009).

12. P. A. Sweet, in IAUsymp.6, B. Lehnert (ed.) (Cambridge University Press, 1958), p. 123.

13. E. N. Parker, J. Geophys. Res. 62, 509 (1957).

14. R. M. Kulsrud, Phys. Plasmas 5, 1599 (1998).

15. J. Goodman and D. Uzdensky, Astrophys. J. 688, 555 (2008).

16. M. Yamada, M. Yamada, R. Kulsrud, and H. Ji, Rev. Modern Phys. 82, 603 (2010).

17. K. Zhang, J. Y. Zhong, J. Q. Wang, X. X. Pei, H. G. Wei, D. W. Yuan, Z. W. Yang, C. Wang, F. Li, B. Han, C. L. Yin, G. Q. Liao, Y. Fang, S. Yang, X. H. Yuan, Y. Sakawa, T. Morita, Z. R. Cao, S. E. Jiang, Y. K. Ding, Y. Kuramitsu, G. Y. Liang, F. L. Wang, Y. T. Li, J. Q. Zhu, J. Zhang, and G. Zhao, High Energy Density Phys. 17, 32 (2015).

18. S. Fujioka, S. Fujioka, Z. Zhang, K. Ishihara, K. Shigemori, Y. Hironaka, T. Johzaki, A. Sunahara, N. Yamamoto, H. Nakashima, T. Watanabe, H. Shiraga, H. Nishimura, and H. Azechi, Sci. Rep. 3, 1170 (2015).

19. J. Shertzer, Phys. Rev. A 39, 3833-3835 (1989).

20. M. G. Dimova, M. S. Kaschiev, and S. I. Vinitsky, J. Phys. B 38, 2337 (2005).

21. L. B. Zhao and P. C. Stancil, J. Phys. B 40, 4347 (2007).

22. Y. P. Kravchenko, M. A. Liberman, and B. Johansson, Phys. Rev. Lett. 77, 619 (1996).

23. S. L. Zeng, S. Zou, and J. Yan, Chin. Phys. Lett. 26, 053202 (2009)

24. J. E. Bailey, T. Nagayama, G. P. Loisel, G. A. Rochau, C. Blancard, J. Colgan, Ph. Cosse, G. Faussurier, C. J. Fontes, F. Gilleron, I. Golovkin, S. B. Hansen, C. A. Iglesias, D. P. Kilcrease, J. J. MacFarlane, R. C. Mancini, S. N. Nahar, C. Orban, J.-C. Pain, A. K. Pradhan, M. Sherrill, and B. G. Wilson, Nature 517, 56 (2015).

25. A. Vourlidas, R. A. Howard, E. Esfandiari, S. Patsourakos, S. Yashiro, and G. Michalek, Astrophys. J. 722, 1522 (2010).

26. E. P. Carley, D. M. Long, J. P. Byrne, P. Zucca, D. S. Bloomfield, J. McCauley, and P. T. Gallagher, Nat. Phys. 9, 811 (2013).

27. D. Yuan and Y. Li, Chin. Phys. B 24, 015204 (2015).

28. X. Liu, Y. T. Li, Y. Zhang, J. Y. Zhong, W. D. Zheng, Q. L. Dong, M. Chen, G. Zhao, Y. Sakawa, T. Morita, Y. Kuramitsu, T. N. Kato, L. M. Chen, X. Lu, J. L. Ma, W. M. Wang, Z. M. Sheng, H. Takabe, Y.-J. Rhee, Y. K. Ding, S. E. Jiang, S. Y. Liu, J. Q. Zhu, and J. Zhang, New J. Phys. 13, 093001 (2011).

29. D. W. Yuan, Y. T. Li, X. Liu, Y. Zhang, J. Y. Zhong, W. D. Zheng, Q. L. Dong, M. Chen, Y. Sakawa, T. Morita, Y. Kuramitsu, T. N. Kato, H. Takabe, Y.-J. Rhee, J. Q. Zhu, G. Zhao, and J. Zhang, High Energy Density Phys. 9, 239 (2013).

30. T. Morita, Y. Sakawa, Y. Kuramitsu, S. Dono, H. Aoki, H. Tanji, T. N. Kato, Y. T. Li, Y. Zhang, X. Liu, J. Y. Zhong, H. Takabe, and J. Zhang, Phys. Plasmas 17, 122702 (2010). 